\title{
PARTICULARITÉS DU DÉVELOPPEMENT DE LA NYMPHE DE FICALBIA METALLICA
}

\author{
Par Henri GALLIARD
}

Dans un article récent, nous avons signalé les particularités concernant l'évolution des nymphes de Mansonioides indianus. Ces nymphes arrivent à donner naissance à des imagos sans jamais avoir été mises

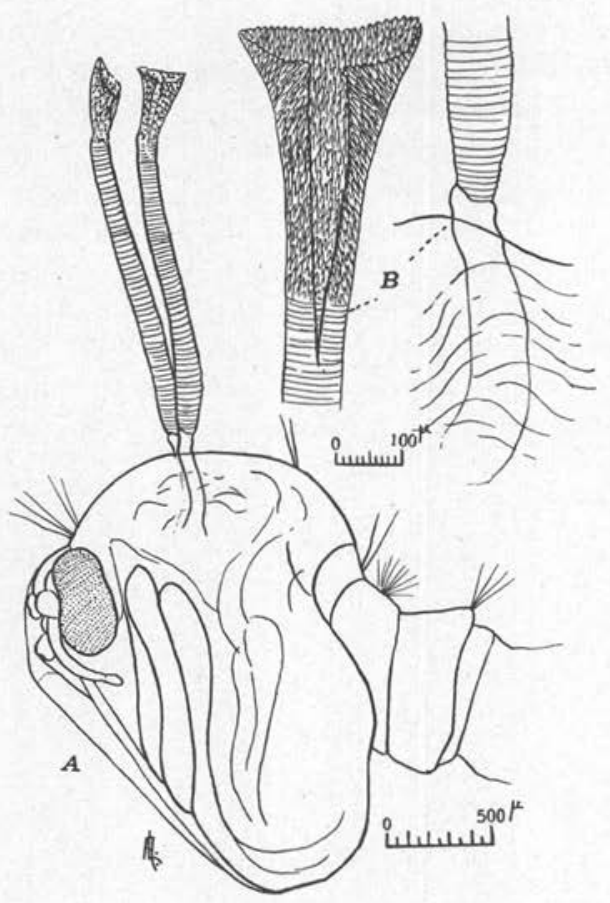

en contact avec des racines où elles auraient pu puiser l'air qui leur est nécessaire. La durée de la phase nymphale dans l'eau distillée est exactement la même que dans les conditions naturelles.

Pour certaines espèces, les nymphes, par suite de circonstances partiansales de Parastrologie, t. XVIII, Nos 4-56. - 1941, p. 245-246. 
culières, peuvent subir des transformations qui ne les empêchent pas de donner naissance à des imagos normaux et viables.

On connait, depuis la description de Iyengar ( $\mathrm{I} 935$ ), la structure très particulière des nymphes de Ficalbia metallica. Nous la reproduisons sur la figure ci-jointe. Les appendices respiratoires sont extrêmement allongés et constitués de deux parties : une partie basale courte et résistante sur laquelle s'insère la portion allongée, dilatée à son extrémité. Cette portion distale est fragile et se brise facilement au point d'union. Il n'est pas rare de récolter de ces nymphes dépourvues d'un ou parfois de leurs deux appendices sans que l'on puisse savoir à quel moment s'est produite la rupture.

Mais nous avons aussi obtenu à plusieurs reprises de ces nymphes mutilées au moment de leur éclosion de la larve. Dans ces conditions, on voit toujours que celles qui sont dépourvues de la partie la plus importante de leur système respiratoire peuvent continuer à vivre et à évoluer normalement. L’imago prend naissance dans les délais normaux.

Il ne s'agit pas d'un défaut de développement, mais, comme nous l'avons dit, d'un traumatisme. Les appendices se brisent au moment où la nymphe se dégage de sa dépouille larvaire; mais ils sont cependant difficiles à retrouver, car le plus souvent ils viennent flotter à la surface. Parfois ils restent pris dans la dépouille larvaire.

Il est certain que les moignons restants sont fonctionnels, car nous avons pu très facilement enlever les appendices respiratoires quelques heures après l'éclosion : les nymphes pouvaient continuer à vivre et à donner naissance à des imagos dans des délais normaux.

Laboratoire de Parasitologie de l'École de Médecine de Hanoi. (Directeur: Professear H.-G. Galliard). 